\title{
DOCKET-LESS RECKON IN METRO'S ADAPTING N.F.C
}

\author{
Saryu Chugh ${ }^{1}$ \\ ${ }^{1}$ Assistant Professor, Chitkara University
}

\begin{abstract}
A modernistic impermanent system empowers a trade of information within the scope of $10 \mathrm{~cm}$ amid devices; an intensification of RFID. It can accurately endeavor while the gadgets are not hopped up by battery. The system is intended for the metro rails which comforts the users in perusing the information by percussing the gadgets to the card readers system at source and will deduct the money after perusing it bis to the destination. The communication materializes in two states; with the mobile device i.e sender is in active state and the other node i.e the receiver is in passive state and second both the devices are in active state i.e in the live condition.
\end{abstract}

Keywords: - N.F.C, RFID, Metro.

\section{INTRODUCTION}

In today's digital era, change is frequent and necessary for agreeable, effective and convenient communication between two or more persons or between two or more electronic devices such as smartphones and other handy devices. N.F.C. is a technology which will fulfill all needs of communication for a portable smartphones without any wired connection between them. The wireless connection is planted or created just by bringing two devices in the range of $10 \mathrm{~cm}$. The communication occurs in two states such as first the mobile device i.e sender is in active state and the other node i.e the receiver is in passive state and second both the devices are in active state i.e in the live condition. Transmission of data is done with the help of electromagnetic field which is nothing but the RFID. We have to bring two devices close to each other and just by giving tag on device the information which is stored in the form of electronic signal is getting transmitted. The N.F.C. is used in many areas such as for making payment, reading information from a smart poster and in ad-hoc for sharing data.

Now a day's very few number of people are using direct cash payment for anything. Everyone is using E-banking, debit cards, credit cards for making payment because it is reliable, convenient and less time consuming. For metro's traveling payment, coupon and card system is existing but for this the customer have to wait into the queue and have to pay cash amount for issuing coupons and card and also sometimes these coupons and cards are not properly working which makes the system little inconvenient. To overcome drawback of traditional card-payment system we are introducing the card-less and user-friendly payment system by N.F.C. In this generation, each person has a smartphone, so by using an application, which is connected to bank server we can make payment directly, just by tapping the smartphone at source first and then destination. Automatically the distance between source and destination is calculated and the respective amount according to distance traveled is deducted from your bank account. This will replace the traditional and orthodox card-payment system by delightful, customer-oriented and timesaving card-less payment system with the help of N.F.C.

\section{LITERATURE REVIEW}

[1]. The automation is thriving on the planet with its clinquant idiosyncrasy and has been excogitate over numerous different advances like barcodes, RFID etc. The article gives the component to defray adopting NFC. It can be further utilized for the association of digital and printed materials. It has numerous different operations like identifications and access control. It has accessible adaptation and adaptable contrasted with different gadgets.

[11]. The apprehensions for the mobile device fabricators with respect to NFC is to be OS free and equipment autonomous, to improve such functionalities NFC stack engineering is volunteered. The engineering exemplifies administrations of OS with reference to NFC stack. It can be servituted on surpassing OS frameworks.

[4]. The intensification of RFID is proposed as NFC which helps in nudge less elucidation. It helps in information trade, nudge less exchanges. Multifarious modes of NFC are ingrained in order to exchange the information.

[6]. The scrutiny of the NFC system is premendiated to be the cardinal matter of contention. It can be influenced by fundamental assaults like listening stealthily.In this strategy, two gadgets are utilized to send arbitrary bits to each other at the aforementioned time. It produces shared keys and symmetric keys for the era of bits which upgrades the security of the framework.

[10] .The accelerated contrivance of utterance is voluntared to be NFC. The innovation works in cell telephones and comparision amongst bluetooth and it can work in shorter extents and security is being dissected. The NFC accredit 
systems has chips inaugurated in it. The framework is dynamic which produces its radio recurrence and different gadgets can be utilized for the information trade. It can be additionally be utilized for the isolating of installments.

[5]. Succeding 12 years of innovation, the integral has proceed the reputation. The framework has advanced to be superior to other gadgets and works prominent and expeditious over some other gadget. It can work at the information rate of 106, 212 and $424 \mathrm{Kbps}$. It can fill in as shared and card copying and so on.

\section{PROPOSED WORK}

The proposed system implements NFC devices in Metro Rail payment. As NFC accord communication between two electronic gadgets. Where each station will have NFC devices which will work equivalent to RFID card reader and one's mobile phone will work alike as RFID card.

Process involves following steps

- Tag mobile phone with NFC device (machine) present at Source Station.

- Which will identifies source station name from database.

- Again, Tag the mobile phone at the destination Station with the particular machine.

- Which will identifies destination station name from database.

- It will calculate the distance between two stations.
- Apply fare as per distance measured between the stations.

- Charges will be deduced from the individuals account.

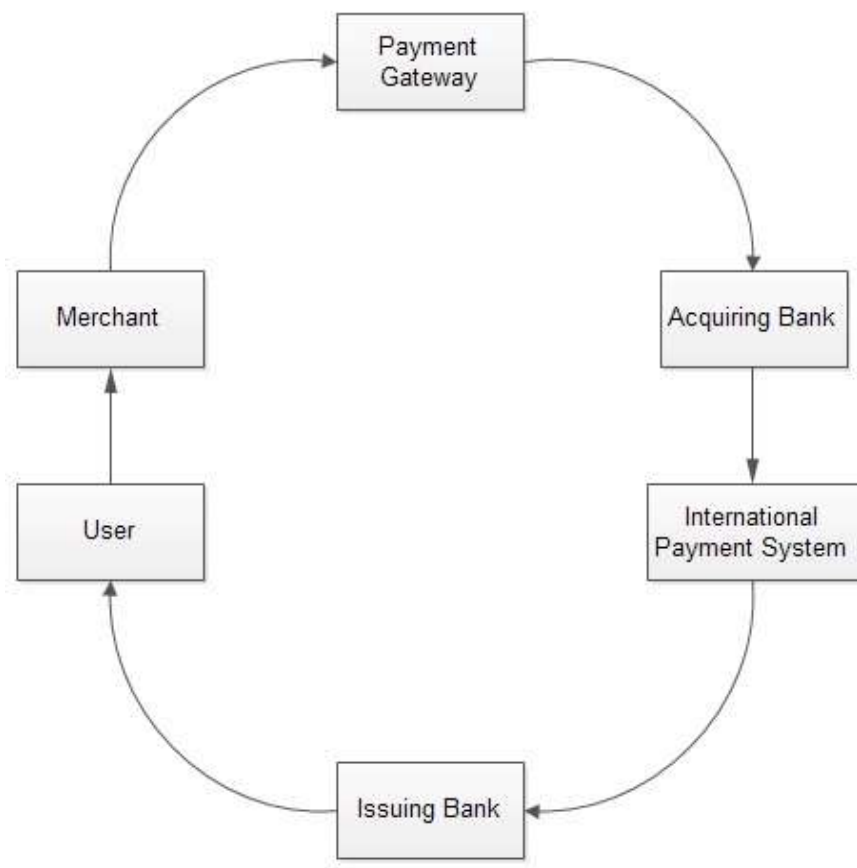

Fig 2: Payment Process

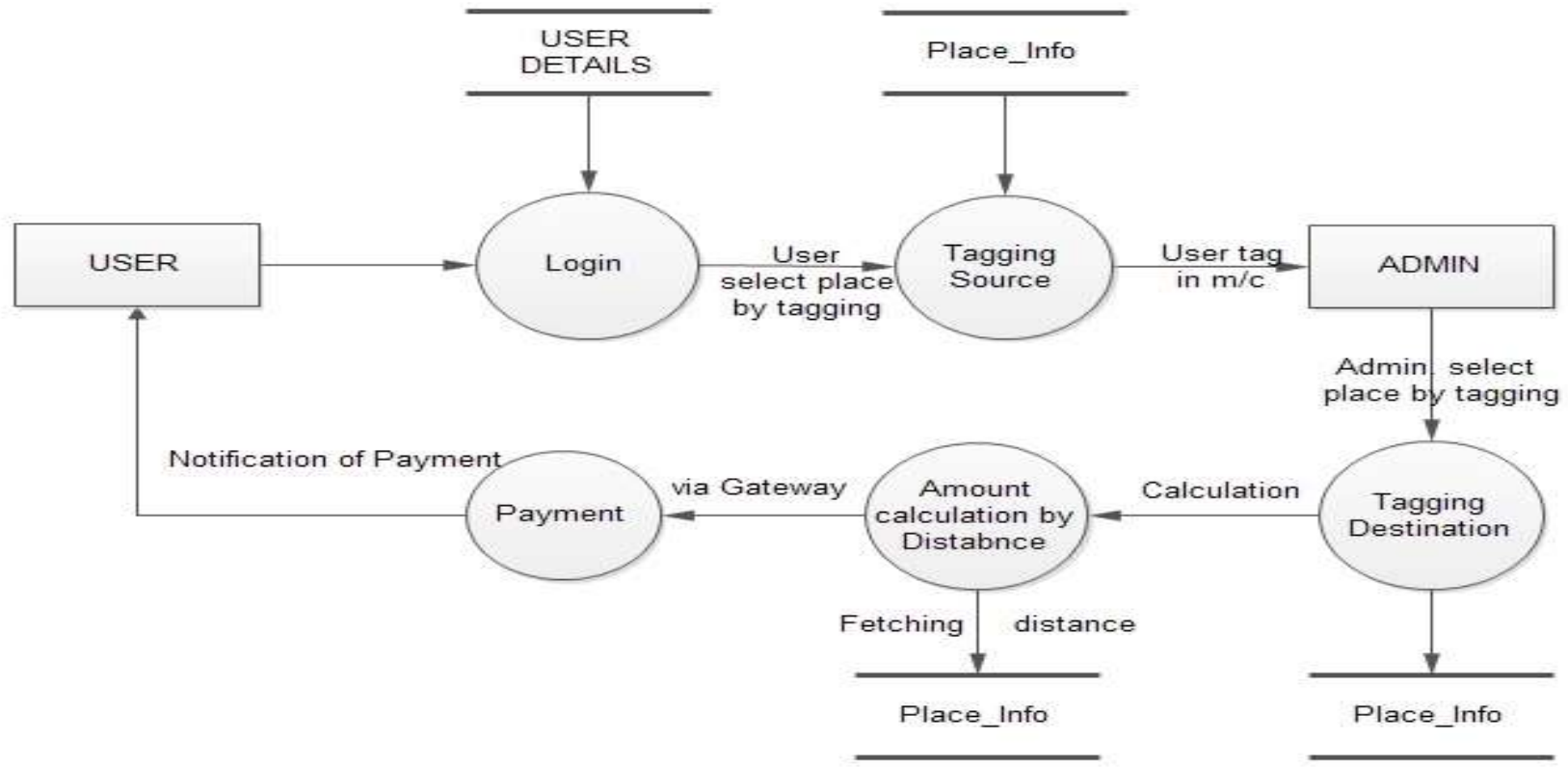

Fig 1: System Process

\section{RESULT AND ANALYSIS}

Scrutinize the methodology deployed using NFC, then the results can be ceased as the NFC can be contemplated as card with which the money will get deducted by percussing it on the source and destination. The framework will deduct the cash from point of view financial balances and the information will be put away in the cloud's database. 


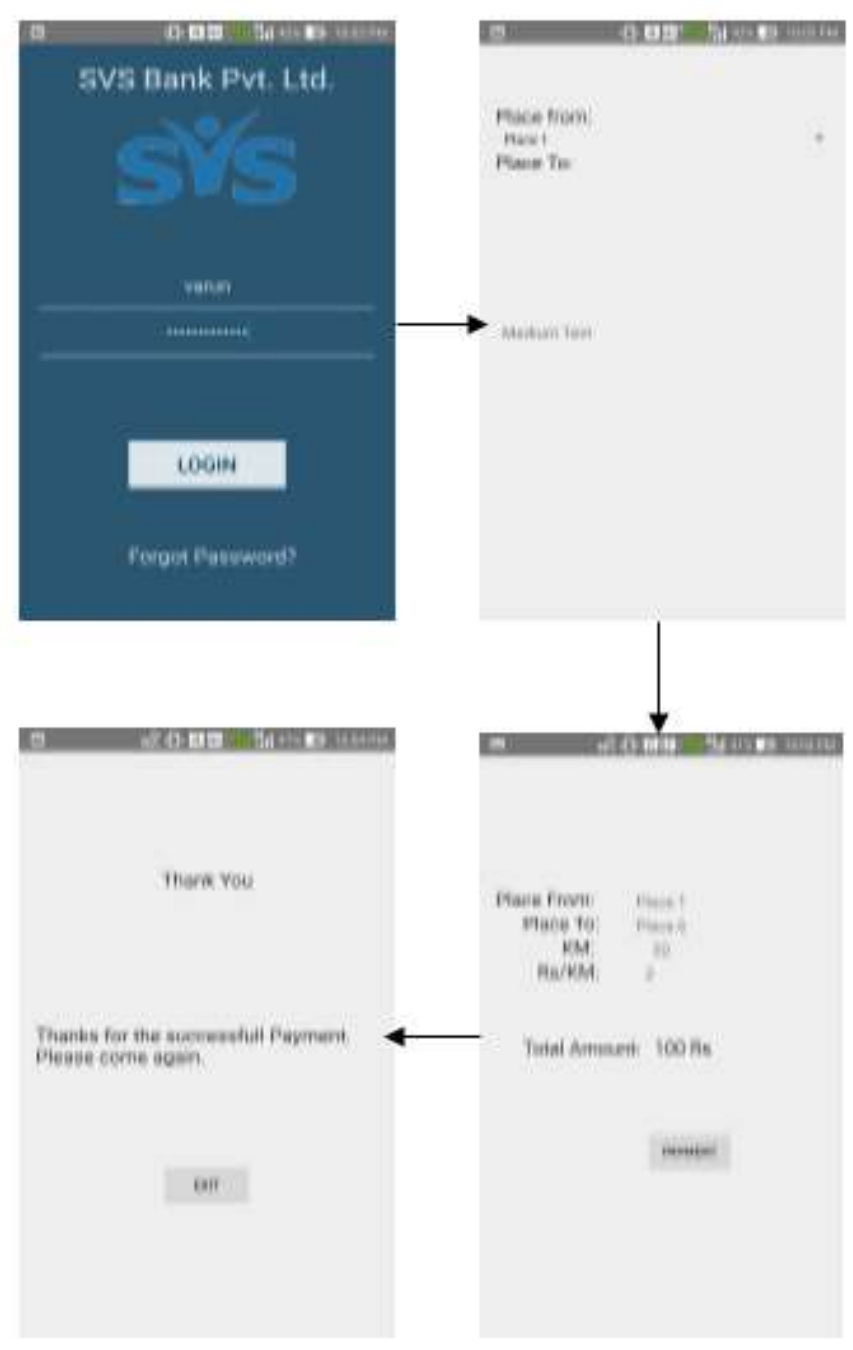

\section{CONCLUSION}

The scrutinized N.F.C has the ability to prosper the expertise, and has the restricted correspondence system which won't roll out clients improvement the destination and source, due to this faulty amount pay will not occurred and security will be sustained. It changes the way of living and transforming everyday tasks, making things facile and more implicit. With addition, it brings well-being setup of longerrange wire-less scheme. Thus, it is very much similar to normal mobile expertise so that user can easily access idea out of it. The future extension of this can be considered with the way to have nexus with the bank systems.

\section{REFERENCES}

[1]. Hiroki Shibuya; Tatsuaki Tsukuda; Hiroko Suzuki; Tadashi Shimizu; Masahiro Dobashi; Shinji Nishizono; Mikio Baba; Hideki Sasaki; Katsushi Terajima, “A wireless charging and near-field communication combination module for mobile applications", IEEE 64th Electronic Components and Technology Conference (ECTC), 2014, pp-763 - 768. [2]. Surya Michrandi Nasution; Emir Mauludi Husni; Aciek Ida Wuryandari, "Prototype of train ticketing application using Near Field Communication (NFC) technology on
Android device", International Conference on System Engineering and Technology (ICSET), 11-12 Sept. 2012.

[3]. Utsav Jambusaria, Neerja Katwala, Dharmeshkumar Mistry, "Secure Smartphone Unlocking Using NFC", International Conference on Advanced Computing Technologies and Applications (ICACTA), Volume 45, 2015, pp.465-469.

[4]. Martin Ebner, Martin Maierhuber, "Near Field Communication - Which Potentials Does NFC Bring for Teaching and Learning Materials?", International Journal of Interactive Mobile Technologies, Vol 7, No 4 (2013).

[5]. J. Choo; J. Ryoo; I. Park; J. Hong; K. Park; J. Lee, “A Novel Multi-loop Tag for Near Field Communication in UHF Band", Asia-Pacific Microwave Conference, 11-14 Dec.2007, pp.1-4.

[6]. Xiao Kun, Luo Lei, "A Novel Mobile Device NFC Stack Architecture", IEEE 11th International Conference on Dependable, Autonomic and Secure Computing, 21-22 Dec. 2013, pp.169 - 173 .

[7]. Gerald Madlmayr, Christian Kantner, and Thomas Grechenig, "Near Field Communication,"Secure Smart Embedded Devices, Platforms and Applications, Springer Press, in press, pp.351-367.

[8]. Akshay Uttama Nambi S.N., Prabhakar T.V., Jamadagni H.S., Kishan Ganapathi, Pramod B.K., Rakesh C.M., and Sanjay Naik R.,"Near Field Communication -Applications and Performance Studies," Wireless Networks and Computational Intelligence, Springer Press, 2012, pp.1-10.

[9]. Kerem OK, Vedat COSKUN, Mehmet N. AYDIN, and Busra OZDENIZCI, "Current Benefits and Future Directions of NFC Services,"Proc. 2010 International Conference on Education and Management Technology^ICEMT 2010^, IEEE Press, 2010, pp. 334 338.

[10]. Rong Jin ; Xianru Du ; Zi Deng ; Kai Zeng, "Practical Secret Key Agreement for Full-Duplex Near Field Communications", IEEE Transactions on Mobile Computing ,Volume:15, Issue: 4 , 18 May 2015, pp. 938 951.

[11]. Yen-Sheng Chen; Shih-Yuan Chen ; Hsueh-Jyh Li, "Analysis of Antenna Coupling in Near-Field Communication Systems ", IEEE Transactions on Antennas and Propagation ,Volume:58, Issue: 10, 01 July 2010 ,pp. 3327 - 3335.

[12]. G. de Meulenaer, F. Gosset, O.-X. Standaert, and O. Pereira, "On the energy cost of communication and cryptography in wireless sensor networks," inProc. IEEE Int. Conf. Wireless Mobile Computer Network Communication, 2008, pp. 580-585.

[13]. S.-H. Wu and C. Yang, "Promoting collaborative mobile payment by using NFC-micro SD technology," inProc. IEEE Int. Conf. Serv. Comput., 2013, pp. 454-461. [14]. Jianwei Niu; Wenfang Song ; Chuang Liu ; Lei Shu; "NECAS: Near field communication system for smartphones based on visible light ", IEEE Wireless Communications and Networking Conference (WCNC), 6-9 April 2014, pp. 2426 - 2431. 
[15]. Roy Want, "Near Field Communication", IEEE Pervasive Computing, vol.10, no. 3, pp. 4-7, July-September 2011.

[16]. Geoffrey Ottoy, Sam Van Den Berge, Jean Pierre Goemaere, Lieven De Strycker, " Measuring the NFC Peerto-Peer Data Rate", European Conference on the Use of Modern Information and Communication Technologies, Vol-302, March 2014, pp- 109-122.

[17]. Max Günther, Bernd Borcher, " Online Banking with NFC-Enabled Bank Card and NFC-Enabled Smartphone", Information Security Theory and Practice. Security of Mobile and Cyber-Physical Systems, Volume 7886, May2013, pp 66-81.

[18]. Gabriele Rescio, Alessandro Leone ; Giovanni Montagna ; Pietro Siciliano," Open and low power near field communication -based platform in healthcare applications ", IEEE SENSORS 2014 Proceedings , 2-5 Nov. 2014, pp .2242-2244 